The Two Phase Drum with the Deepest Bass Note

Steven J. Cox

October, 1990

TR90-28 



\title{
THE TWO PHASE DRUM WITH THE DEEPEST BASS NOTE
}

\author{
Steven J. Cox
}

Mathematical Sciences Department, Rice University, Houston, TX 77251. Research supported by Defense Advanced Research Projects Agency Contract F49620-87-C0065 and Air Force Office of Scientific Research Grant 89-0363.

\section{Introduction}

On an open bounded connected set $\Omega \subset \mathbf{R}^{2}$ we consider

$$
-\Delta u=\lambda \rho u, \quad \text { in } \Omega,\left.\quad u\right|_{\partial \Omega}=0
$$

where the density $\rho$ is chosen from the class

$$
\begin{array}{r}
a d=\{\alpha \chi+\beta(1-\chi): \chi \text { is the characteristic } \\
\text { function of a subset of } \Omega \text { with area } \gamma\} .
\end{array}
$$

Here $\alpha$ and $\beta$ are the known (constant) densities of two given materials in respective volume fractions $\gamma /|\Omega|$ and $1-\gamma /|\Omega|$. We assume that $\alpha<\beta$. Krein [7] considered the minimization of $\rho \mapsto \lambda_{1}(\rho)$, the least eigenvalue of (1.1), over $a d$ in the case where $\Omega$ is a disc. He argued that the minimum was obtained by placing the material of greater density in a concentric disc, the remaining annulus to then be filled with the material of lesser density. Friedland [2] found that $\rho \mapsto \lambda_{1}(\rho)$ indeed attains its minimum over $a d$ for any open bounded connected $\Omega$. The question of uniqueness has yet to be resolved. As $\rho \mapsto 1 / \lambda_{1}(\rho)$ is convex and functions and their reciprocals share level sets one finds $\left\{\rho \in L^{\infty}: \lambda_{1}(\rho) \geq c\right\}$ to be convex for each $c \in \mathbf{R}$. Quasiconcave functions need not possess unique minimizers. Denoting a minimizer by $\check{\rho}$, Cox and McLaughlin [1] established the existence of an $\ell>0$ for which

$$
\check{\rho}(x)= \begin{cases}\alpha & \text { if } \breve{u}(x)<\ell \\ \beta & \text { if } \breve{u}(x)>\ell,\end{cases}
$$

for almost every $x \in \Omega$, where $\breve{u}$ is an eigenfunction corresponding to $\lambda_{1}(\check{\rho})$. This $\breve{u}$ is a positive superharmonic element of the Sobolev space $W^{2, p}(\Omega)$ for each $p<\infty$ and satisfies (1.1) almost everywhere. The optimal distribution of mass is completely determined by its level set,

$$
\Gamma \equiv \check{u}^{\leftarrow}(\ell)=\{x \in \Omega: \check{u}(x)=\ell\}
$$

This work is an investigation of the shape, smoothness, and computation of the interface $\Gamma$. The success of this study derives from the fact that the optimality condition (1.2) overdetermines the equilibrium equation (1.1) to such a degree that one can read off important information about the optimal design. In particular, it will be shown under natural geometric assumptions on $\Omega$ that $\Gamma$ is analytic. The 
geometric hypotheses are needed to reach $\Gamma \in C^{\mathbf{1}}$, from which point the standard regularity theory applies. The use of rearrangements in $\S 2$ suggests a simple approach to the computation of $\Gamma$. An algorithm is formulated in $\S 3$ with the results of its use discussed in $\S 4$.

\section{Symmetry and Regularity}

An open set $\Omega$ in the plane is said to be Steiner symmetric about the line $L$ when (i) it coincides with its reflection across $L$ and (ii) its intersection with each line perpendicular to $L$ is either connected or empty. The Steiner symmetrization of $\Omega$ with respect to the line $L$ is the set $\Omega^{L}$ such that, for every line $L^{\perp}$ perpendicular to $L$, either $L^{\perp} \cap \Omega=\emptyset$ and $L^{\perp} \cap \Omega^{L}=\emptyset$, or $L^{\perp} \cap \Omega \neq \emptyset$ and $L^{\perp} \cap \Omega^{L}$ is a closed segment with center on $L$ and $\left|L^{\perp} \cap \Omega^{L}\right|=\left|L^{\perp} \cap \Omega\right|$. Observe that $\Omega=\Omega^{L}$ if and only if $\Omega$ is Steiner symmetric with about $L$.

This symmetrization induces a corresponding rearrangement of functions defined on $\Omega$. In particular, for $u: \Omega \rightarrow \mathbf{R}$ we consider $u^{L}: \Omega^{L} \rightarrow \mathbf{R}$,

$$
u^{L}(x)=\sup \left\{c \in \mathbf{R}: x \in\left(u^{\leftarrow}[c,+\infty)\right)^{L}\right\} .
$$

Of the numerous properties enjoyed by $u \mapsto u^{L}$ recall (Kawohl [5]),

$$
\begin{gathered}
\left(u^{L}\right)^{\leftarrow}(c,+\infty) \text { is Steiner symmetric about } L \forall c \in \mathbf{R}, \\
\text { if } u \in L^{1}(\Omega) \text { then }\left|u^{\leftarrow}[c,+\infty)\right|=\left|\left(u^{L}\right)^{\leftarrow}[c,+\infty)\right| \quad \forall c \in \mathbf{R}, \\
\text { if } u, v, w \in L^{1}(\Omega) \text { then } \int_{\Omega} u v w d x \leq \int_{\Omega^{L}} u^{L} v^{L} w^{L} d x, \quad \text { and } \\
\text { if } u \in H_{0}^{1} \text { then } \int_{\Omega}|\nabla u|^{2} d x \geq \int_{\Omega^{L}}\left|\nabla u^{L}\right|^{2} d x .
\end{gathered}
$$

On assuming $\Omega$ to be Steiner symmetric with respect about $L$ and denoting, as above, the minimizer of $\rho \mapsto \lambda_{1}(\rho)$ by $\check{\rho}$, we find, as a consequence of (2.2) that $\check{\rho}^{L} \in a d$. Moreover, on rearranging the $\breve{u}$ of (1.2) we find, as a consequence of Rayleigh's Principle, (2.3-4), and $\Omega=\Omega^{L}$, that

$$
\lambda_{1}(\check{\rho})=\frac{\int_{\Omega}|\nabla \breve{u}|^{2} d x}{\int_{\Omega} \check{\rho} \check{u}^{2} d x} \geq \frac{\int_{\Omega}\left|\nabla \check{u}^{L}\right|^{2} d x}{\int_{\Omega} \check{\rho}^{L}\left|\check{u}^{L}\right|^{2} d x} \geq \lambda_{1}\left(\check{\rho}^{L}\right) .
$$

Hence, we may assume without loss that $\check{\rho}=\check{\rho}^{L}$ and $\breve{u}=\breve{u}^{L}$. It now follows immediately from (2.1) that

Theorem 1. If $\Omega$ is Steiner symmetric about a line $L$ through the origin then

(i) $\breve{\rho}^{\leftarrow}(\beta)$ is Steiner symmetric about $L$.

(ii) $(\xi \cdot x)(\xi \cdot \nabla \check{u}(x)) \leq 0$ for each $x \in \Omega$ where $\xi$ is normal to $L$ at the origin.

When $\Omega$ is a disk this result vindicates Krein's belief in a circular $\Gamma$ and radial $\breve{u}$. In addition, it suggests an approach to the question of the regularity of $\Gamma$. This 
set is smooth except at those points where $\nabla \check{u}$ vanishes. For sufficiently symmetric domains one may expect $\breve{u}$ to be stationary nowhere save the center of symmetry.

Theorem 2. If $\Omega$ is Steiner symmetric about two independent lines then $\Gamma$ is analytic.

Proof: Without loss we assign the center of symmetry to be the origin in our $\left(x_{1}, x_{2}\right)$ coordinate system and assume that $\Omega$ is Steiner symmetric about the $x_{2}$ axis. We shall establish that $\Gamma$ is (a) rectifiable, (b) $C^{1}$, and finally (c) analytic.

(a) Every bounded planar set that is Steiner symmetric about two independent lines has a rectifiable boundary. The proof of this elementary fact will later facilitate the construction of an explicit bound on the length of $\Gamma$. Denote by $L_{1}$ the $x_{2}$ axis and by $L_{2}$ the second line of Steiner symmetry. Let $\phi$ denote the angle that $L_{2}$ makes with the $x_{1}$ axis and, for convenience, assume $0 \leq \phi \leq \pi / 2$. We consider $\Gamma^{\prime}$, the restriction of $\Gamma$ to the sector lying between $L_{1}$ and $L_{2}$, i.e.,

$$
\Gamma^{\prime} \equiv \Gamma \cap\{(r, \theta): 0 \leq r, \phi \leq \theta \leq \pi / 2\}
$$

By symmetry $\Gamma$ can be recovered by reflecting $\Gamma^{\prime}$ across $L_{1}$ and $L_{2}$ no more than seven times. It remains then to show that $\Gamma^{\prime}$ is rectifiable. Given $\left(x_{1}, x_{2}\right) \in \Gamma^{\prime}$, convexity of $\check{u}^{\leftarrow}(\ell,+\infty)$ with respect to lines perpendicular to $L_{1}$ and $L_{2}$ requires that

$$
\Gamma^{\prime} \subset\left(x_{1}, x_{2}\right)+\{(r, \theta): 0 \leq r, \pi / 2-\phi \leq \theta \leq \pi, \text { or } 3 \pi / 2-\phi \leq \theta \leq 2 \pi\}
$$

see Figure 1. It follows that $\Gamma^{\prime}$ is the graph of a monotone (Lipschitz in case $\phi \neq 0$ ) function and hence rectifiable.

(b) The gradient of $\breve{u}$ can vanish nowhere save the origin. Consequently, every level set of $\check{u}$ is as smooth as $\check{u}$ itself, i.e., $C^{1}$.

We denote the preimage $\breve{u}^{\leftarrow}(0, c)$ by $\Omega_{c}$ and its characteristic function by $\chi^{c}$. As $\Omega_{\ell}$ has been shown to be of finite perimeter we may exploit the fact that $\nabla \chi^{\ell}$ is a vector Radon measure supported on $\Gamma \cup \partial \Omega$. The subscript $x_{1}$ will denote partial differentiation with respect to $x_{1}$. In the interest of showing $\breve{u}_{x_{1}}$ to be subharmonic when $x_{1}>0$ we select a nonnegative $\phi \in C_{0}^{\infty}\left(x \in \Omega: x_{1}>0\right)$ and compute

$$
\begin{aligned}
\int_{\Omega} \check{u}_{x_{1}} \Delta \phi d x & =-\int_{\Omega} \check{u} \Delta \phi_{x_{1}} d x=\int_{\Omega} \nabla \check{u} \cdot \nabla \phi_{x_{1}} d x \\
& =\check{\lambda}_{1} \int_{\Omega} \check{\rho} \check{u} \phi_{x_{1}} d x=-\check{\lambda}_{1} \int_{\Omega}(\check{\rho} \check{u})_{x_{1}} \phi d x \\
& =-\check{\lambda}_{1} \int_{\Omega} \check{\rho} \check{u}_{x_{1}} \phi d x-\check{\lambda}_{1} \int_{\Omega} \check{\rho}_{x_{1}} \check{u} \phi d x
\end{aligned}
$$

By part (ii) of Theorem $1, \breve{u}_{x_{1}} \leq 0$ on the support of $\phi$, and so the first term is nonnegative. Regarding the second, note that $\check{\rho}_{x_{1}}=\left(\alpha \chi^{\ell}+\beta\left(1-\chi^{\ell}\right)\right)_{x_{1}}=$ 
$(\alpha-\beta) \chi_{x_{1}}^{\ell}$. Now $\chi_{x_{1}}^{\ell}$ is a measure supported on $\Gamma \cup \partial \Omega, \breve{u}=\ell$ on $\Gamma$ and $\phi$ has compact support in $\Omega$, so the second term is precisely

$$
\check{\lambda}_{1}(\beta-\alpha) \ell \int_{\Gamma} \phi \chi_{x_{1}}^{\ell} \text {. }
$$

We proceed to show that $\int_{\Gamma} \phi \chi_{x_{1}}^{\ell} \geq 0$. That $\breve{u} \in C^{\infty}\left(\Omega_{c}\right)$ for $c<\ell$ follows from the fact that $\breve{u}$ satisfies a constant coefficient elliptic partial differential equation there. Consequently, Sard's theorem reveals a sequence $\left\{\varepsilon_{j}\right\}_{j=1}^{\infty}, \varepsilon_{j}<0, \varepsilon_{j} \uparrow 0$ such that each $\ell-\varepsilon_{j}$ is a regular value of $\check{u}$. As $\Omega_{\ell-\varepsilon_{j}}$ has a smooth boundary the divergence theorem takes the form

$$
\begin{aligned}
\chi_{x_{1}}^{\ell-\varepsilon_{j}} & =\nu_{1}^{\ell-\varepsilon_{j}}\left|\nabla \chi^{\ell-\varepsilon_{j}}\right| \\
& =\frac{-\check{u}_{x_{1}}}{|\nabla \check{u}|} d \mathcal{H}
\end{aligned}
$$

where $\nu_{1}^{\ell-\varepsilon_{j}}$ is the $x_{1}$ component of the interior normal to $\Omega_{\ell-\varepsilon_{j}}$ and $d \mathcal{H}$ denotes one-dimensional Hausdorff measure. Integrating by parts on $\Omega_{\ell-\varepsilon_{j}}$ gives

$$
\begin{aligned}
\int_{\Omega_{\ell-\varepsilon_{j}}} \phi_{x_{1}} d x & =-\int_{\partial \Omega_{\ell-\varepsilon_{j}}} \phi \chi_{x_{1}}^{\ell-\varepsilon_{j}} \\
& =\int_{\partial \Omega_{\ell-\varepsilon_{j}}} \phi \frac{\check{u}_{x_{1}}}{|\nabla \check{u}|} d \mathcal{H} \leq 0 .
\end{aligned}
$$

Hence, as $\varepsilon_{j} \rightarrow 0$,

$$
\int_{\Omega_{\ell-\ell_{j}}} \phi_{x_{1}} d x \rightarrow \int_{\Omega_{\ell}} \phi_{x_{1}} d x=-\int_{\Gamma} \phi \chi_{x_{1}}^{\ell} \leq 0
$$

As a result,

$$
\int_{\Omega} \check{u}_{x_{1}} \Delta \phi d x \geq 0 \text { for all nonnegative } \phi \in C_{0}^{\infty}\left(x \in \Omega: x_{1}>0\right) \text {. }
$$

As $\check{u}_{x_{1}}$ is nonpositive and subharmonic in $\left\{x \in \Omega: x_{1}>0\right\}$ we conclude from the maximum principle that it must be either strictly negative or vanish identically there. As the latter would require $\check{u}$ itself to vanish on all of $\Omega$ we find that $\check{u}_{x_{1}}<0$ on $\left\{x \in \Omega: x_{1}>0\right\}$. By symmetry with respect to $L_{1}$ we have $\breve{u}_{x_{1}}>0$ on $\left\{x \in \Omega: x_{1}<0\right\}$, and so $\check{u}_{x_{1}} \neq 0$ on $\Omega \backslash L_{1}$. Repeating this argument with $L_{2}$ rather than $L_{1}$ yields $\nabla \check{u} \cdot \xi \neq 0$ on $\Omega \backslash L_{2}$, where $\xi$ is a unit vector orthogonal to $L_{2}$. Combining these observations produces $\nabla \breve{u} \neq 0$ on $\Omega \backslash\{0\}$. In particular, $\ell$ is a regular value of $\check{u}$, and hence $\Gamma$ is a closed one-dimensional submanifold of $\Omega$ with regularity that of $\check{u}$, i.e., $C^{\mathbf{1}}$.

(c) The function $v \equiv \breve{u}-\ell$ satisfies a constant coefficient elliptic problem on each side of the $C^{1}$ curve $\Gamma$ on which it vanishes but possess a nonzero normal derivative. 
In particular, $v \in C^{2}\left(\Omega_{\ell} \cup \Gamma\right) \cap C^{2}\left(\left(\Omega \backslash \Omega_{\ell}\right) \cup \Gamma\right) \cap C^{1}(\Omega)$ satisfies

$$
\begin{array}{rll}
\Delta v+\check{\lambda}_{1} \beta v+\check{\lambda}_{1} \beta \ell=0 & \text { in } \quad & \Omega \backslash \Omega_{\ell} \\
\Delta v+\check{\lambda}_{1} \alpha v+\check{\lambda}_{1} \alpha \ell=0 & \text { in } & \Omega_{\ell} \\
v=0, \quad \frac{\partial v}{\partial \nu} \neq 0 & \text { on } & \Gamma .
\end{array}
$$

One now constructs a parametrization of $\Gamma$ that by (2.5) forms one component of the solution of a quasilinear elliptic system with analytic coefficients and coercive boundary conditions. From this it follows, see Kinderlehrer et. al. [6, 3.1'], that $\Gamma$ is analytic.

When $\Omega$ is a regular $n$-sided polygon one can obtain explicit bounds on the length of the interface, denoted $\Gamma_{n}$ for convenience. As $\Gamma_{n}$ encloses a region of area $|\Omega|-\gamma$ the isoperimetric equality renders $2 \sqrt{\pi(|\Omega|-\gamma)} \leq\left|\Gamma_{n}\right|$. Regarding an upper bound the argument that lead to Fig. 1 implies that $\Gamma^{\prime}$ is shorter than one half the perimeter of its bounding parallelogram. This observation, after some elementary plane geometry, produces

$$
2 \sqrt{\pi(|\Omega|-\gamma)} \leq\left|\Gamma_{n}\right| \leq 4 \sqrt{n(|\Omega|-\gamma)}\left(\frac{1-\cos (\pi / n)}{\sin ^{3 / 2}(\pi / n) \cos ^{1 / 2}(\pi / n)}\right)
$$

The upper bound is sharp in the sense that it decreases to the lower bound as $n \rightarrow \infty$. Evaluating these bounds with $n=4$ and $|\Omega|=1$, the case to considered in $\S 4$, produces $3.545 \sqrt{1-\gamma} \leq\left|\Gamma_{4}\right| \leq 4.686 \sqrt{1-\gamma}$.

From the proof of Theorem 2 it is obvious that the two directions of symmetry guarantee the absence of stationary points of $\check{u}$ at points other than the center. That two directions are actually necessary is perhaps most easily seen by considering a barbell shaped drum, i.e., a drum composed of two disks joined by a long thin rectangle. One expects $\breve{u}$ to be large in the disks and small in the rectangle, and consequently to admit a local maximum in each disk and a saddle in the rectangle. One can now tune $\gamma$ so that $\Gamma$ contains the saddle point.

Friedland [2] actually found that each eigenvalue of (1.1) attains its minimum on ad. In [1] it was shown that the interface in the two phase drum with the deepest second eigenvalue is the level set of a second eigenfunction in each of its nodal domains. To establish regularity of this interface one first needs to establish symmetry of the nodal domains. This issue also arises in Friedland's analysis [3] of the associated problem on surfaces; Given a compact two-dimensional Riemannian manifold $\mathcal{M}$ find that manifold, conformal to $\mathcal{M}$ (with the ratio of their metrics lying between $\alpha$ and $\beta$ ) and of prescribed area, with the least first nonzero eigenvalue. When $\mathcal{M}$ is a sphere Friedland finds in [3] the analog of Krein's solution, i.e., the metric is $\beta$ on two antipodal caps and $\alpha$ in the complementary band about the equator. 


\section{An Algorithm}

Upon discretizing $\Omega=\cup_{i} \triangle_{i}: i=1, \ldots, n$ we approximate the membrane's displacement by members of the $n$-dimensional space $W_{n} \subset W_{0}^{1,2}(\Omega)$ and its density by members of $a d_{n} \subset a d$. The choice of $W_{n}$ will be determined by the particular discretization while $a d_{n}$ will always correspond to those piecewise constant functions that are either identically $\alpha$ or $\beta$ on individual $\triangle_{i}$.

We address the computation of $\breve{\rho}_{n}$, the minimizer of $\rho \mapsto \lambda_{1}(\rho)$ over $a d_{n}$ where $\lambda_{1}(\rho)$ is now the least eigenvalue of

$$
K u=\lambda M(\rho) u
$$

Here $K$ and $M(\rho)$ are the respective stiffness and mass matrices that follow from ones choice of $W_{n}$. Recalling that $\check{\rho}_{n}$ and its corresponding eigenvector, $\check{u}_{n}$, satisfy

$$
\mathcal{R}\left(\check{\rho}_{n}, \check{u}_{n}\right)=\inf _{\rho \in a d_{n}} \inf _{u \in W_{n}} \mathcal{R}(\rho, u), \quad \mathcal{R}(\rho, u)=\frac{u^{T} K u}{u^{T} M(\rho) u}
$$

we propose a simple alternating search. From $\left(\rho^{k}, u^{k}\right) \in a d_{n} \times W_{n}$ choose

1. $u^{k+1}$ to minimize $u \mapsto \mathcal{R}\left(\rho^{k}, u\right)$ over $W_{n}$.

2. $\rho^{k+1}$ to minimize $\rho \mapsto \mathcal{R}\left(\rho, u^{k+1}\right)$ over $a d_{n}$.

3. If $\rho^{k+1} \neq \rho^{k}$ then repeat.

Here $u^{k+1}$, the first eigenvector of the discrete drum of density $\rho^{k}$, is approximated via Sorensen's implicitly restarted Arnoldi method [8]. The $j$ th step of the Arnoldi method produces the partial factorization

$$
K V_{j}-M\left(\rho^{k}\right) V_{j} H_{j}=r_{j} e_{j}^{T}
$$

where $H_{j}$ is Hessenberg and $e_{j}$ is the last column in the identity matrix. Unlike the standard Arnoldi method where the column dimensions of $H_{j}$ and $V_{j}$ increase with $j$ this variant succeeds in capping these dimensions by a value on the order of the number of desired eigenvalues. The norm of the residual $r_{j}$ indeed approaches zero as $j$ becomes large and hence the eigenvalues of $H_{j}$ approach those of $\left(K, M\left(\rho^{k}\right)\right)$ while the image of $H_{j}$ 's eigenvectors under $V_{j}$ approach the eigenvectors of $\left(K, M\left(\rho^{k}\right)\right)$.

Now $\rho^{k+1}$ is nothing but a rearrangement of $\rho^{k}$. It takes $\alpha$ below and $\beta$ above a level set of $u^{k+1}$, so chosen to respect the constraint $\left|\left\{x \in \Omega: \rho^{k+1}(x)=\alpha\right\}\right|=\gamma$. We implement this as follows

2.1 Compute $\bar{u}_{i}$, the average of $u^{k+1}$ over $\triangle_{i}$.

2.2 Find an $\ell$ for which $\left|\left\{i: \bar{u}_{i}<\ell\right\}\right|\left|\triangle_{i}\right| \approx \gamma$.

2.3 Set

$$
\rho^{k+1}\left(\triangle_{i}\right)= \begin{cases}\alpha, & \text { if } \bar{u}_{i}<\ell \\ \beta, & \text { if } \bar{u}_{i}>\ell\end{cases}
$$


In step 2.2 we have followed the suggestion of Goodman et. al. [4] in using the Quickfind algorithm of Hoare [9].

Clearly, steps 1 and 2 yield a numerically stable procedure, i.e.,

$$
0 \leq \mathcal{R}\left(\rho^{k+1}, u^{k+1}\right) \leq \mathcal{R}\left(\rho^{k}, u^{k+1}\right) \leq \mathcal{R}\left(\rho^{k}, u^{k}\right)
$$

In addition, if $(\rho, u)$ is a fixed point of the algorithm then necessarily, $u$ is a first eigenvector of $(K, M(\rho))$ and $\rho$ changes phase across a level set of $u$. It is not hard to see in the 1 -dimensional case, e.g., $\Omega=(0,1)$, that these conditions are actually sufficient, and hence that stationary points of our algorithm are minimizers. In particular, if $\bar{u}$ is the first eigenfunction of the string with density

$$
\bar{\rho}(x)= \begin{cases}\alpha & \text { if } \bar{u}(x)<\ell, \\ \beta & \text { if } \bar{u}(x)>\ell\end{cases}
$$

for some $\ell$, then in fact $\bar{\rho}$ must be $\beta$ on an interval $(a, b)$, for $\bar{u}$ can not admit a positive local minimum. Matching conditions at $a$ and $b$ then require that

$$
a \sqrt{\lambda_{1}(\bar{\rho}) \alpha}=(1-b) \sqrt{\lambda_{1}(\bar{\rho}) \alpha}+m \pi
$$

for some integer $m$. But $\left|(a+b-1) \sqrt{\lambda_{1}(\bar{\rho}) \alpha}\right|<\sqrt{\lambda_{1}(\bar{\rho}) \alpha}<\sqrt{\lambda_{1}(\alpha) \alpha}=\pi$, so $m=0$, i.e., $a=1-b$. With the fact that $b-a=1-\gamma$ this gives $a=\gamma / 2$ and $b=1-\gamma / 2$ in agreement with Krein.

In $\mathbf{R}^{2}$ such an argument is not available. What can be said is that $\rho \mapsto \lambda_{1}(\rho)$ is a quasiconcave function over the convex hull of $a d$. As a result, regardless of the algorithm we choose, we can not be assured of reaching a global minimum.

\section{Numerical Results}

The algorithm was tested on a square drum with bilinear elements approximating its transverse displacement. A random number generator was used to construct each initial mass distribution. Regardless however of the initial configuration, the algorithm successfully terminated at the same drum after fewer than twenty iterations. The result on a 256 -by-256 grid with $\alpha=1, \beta=9$, and $\gamma=1 / 2$ is depicted in Figure 2. The least eigenvalue of this drum is 2.325 compared with $2 \pi^{2} / 5 \approx 3.948$ produced by the homogeneous drum of the same mass. The deepest bass note was found in [1] to be a continuous strictly increasing function of $\gamma$. Figure 3 provides a comparison of the least eigenvalue of the homogeneous drum of mass $1 \gamma+9(1-\gamma)$ and the least eigenvalue of the two phase drum of densities 1 and 9 in volume fractions $\gamma$ and $1-\gamma$ produced by the algorithm of $\S 3$. These results are easily interpreted in light of the optimality conditions, (1.2). In particular, adding low density material to the drum should produce little change in the deepest bass note for the optimal design relegates such material to a neighborhood of the drum's boundary where the displacement is small, hence the negligible slope near $\gamma=0$ in Fig. 3. Just the opposite is true when the high density material is introduced, for the optimal design pushes it to the drum's center where the displacement is greatest 
and so produces a marked decrease in fundamental tone, hence the large slope near $\gamma=1$ in Fig. 3 .

On applying this algorithm to the minimization of the drum's higher frequencies convergence was observed for only the second eigenvalue. Hence, recalling the remarks made at the close of $\S 2$, the numerical scheme breaks down at the point at which the analysis also fails, the culprit in each case being an insufficient number of nodal domains. Regarding the minimization of the second eigenvalue, by judicious choice of starting point the algorithm could be made to rest at one of four possible mass distributions. These four consist of two pair, the elements of a pair being rotations of one another by $\pi / 2$. Figures 4 and 5 give the two distinct rest points on a 200-by-200 grid with again $\alpha=1, \beta=9$, and $\gamma=1 / 2$. The nodal line of the associated second eigenfunction is in each case indicated by dashes. The second eigenvalue of the drums in Figures 4 and 5 are respectively 5.795 and 5.665 compared with $\pi^{2} \approx 9.869$ produced by the homogeneous drum of the same mass. Recalling that a drum's second eigenvalue is the first eigenvalue of each of its nodal domains and that the first eigenvalues of the unit mass homogeneous rectangle with sides 1 and $1 / 2$ and the right triangle with sides 1,1 , and $\sqrt{2}$ coincide, this example suggests that among homogeneous rectangular and triangular drums of equal mass and first eigenvalue, the triangle achieves the deeper bass note when another phase in prescribed fraction is introduced.

\section{References}

[1] S. J. Cox and J. R. McLaughlin, Extremal eigenvalue problems for composite membranes I \& II, Applied Math. \& Opt. 22, 1990, pp. 153-167, 169-187.

[2] Friedland, S., Extremal eigenvalue problems defined for certain classes of functions, Arch. Rat. Mech. Anal., 67(1), 1977, pp. 73-81.

[3] Friedland, S., Extremal eigenvalue problems defined on conformal classes of compact Riemannian manifolds, Comment. Math. Helvetici 54, 1979, pp. 494-507.

[4] Goodman, J., Kohn, R.V., Reyna, L., Numerical study of a relaxed variational problem from optimal design, Computer Methods in Appl. Mech. \& Eng. 57, 1986, pp. 107-127.

[5] Kawohl, B., Rearrangements and Convexity of Level Sets in PDE, Springer Verlag, New York, 1985.

[6] Kinderlehrer, D., Nirenberg, L., and Spruck, J., Regularity in free boundary problems I, Journal d'Analyse Mathématique 34, 1978, pp. 86-119.

[7] Krein, M.G., On certain problems on the maximum and minimum of characteristic values and on the Lyapunov zones of stability, AMS Translations Ser. 2(1), 1955, pp. 163-187.

[8] Sorensen, D., Implicit application of polynomial filters in a $k$-step Arnoldi method, Rice Univ., Dept. of Math. Sci. Tech. Report TR-90-27.

[9] Wirth, N., Algorithms and Data Structures = Programs, Prentice-Hall, Englewood Cliffs, NJ, 1976. 


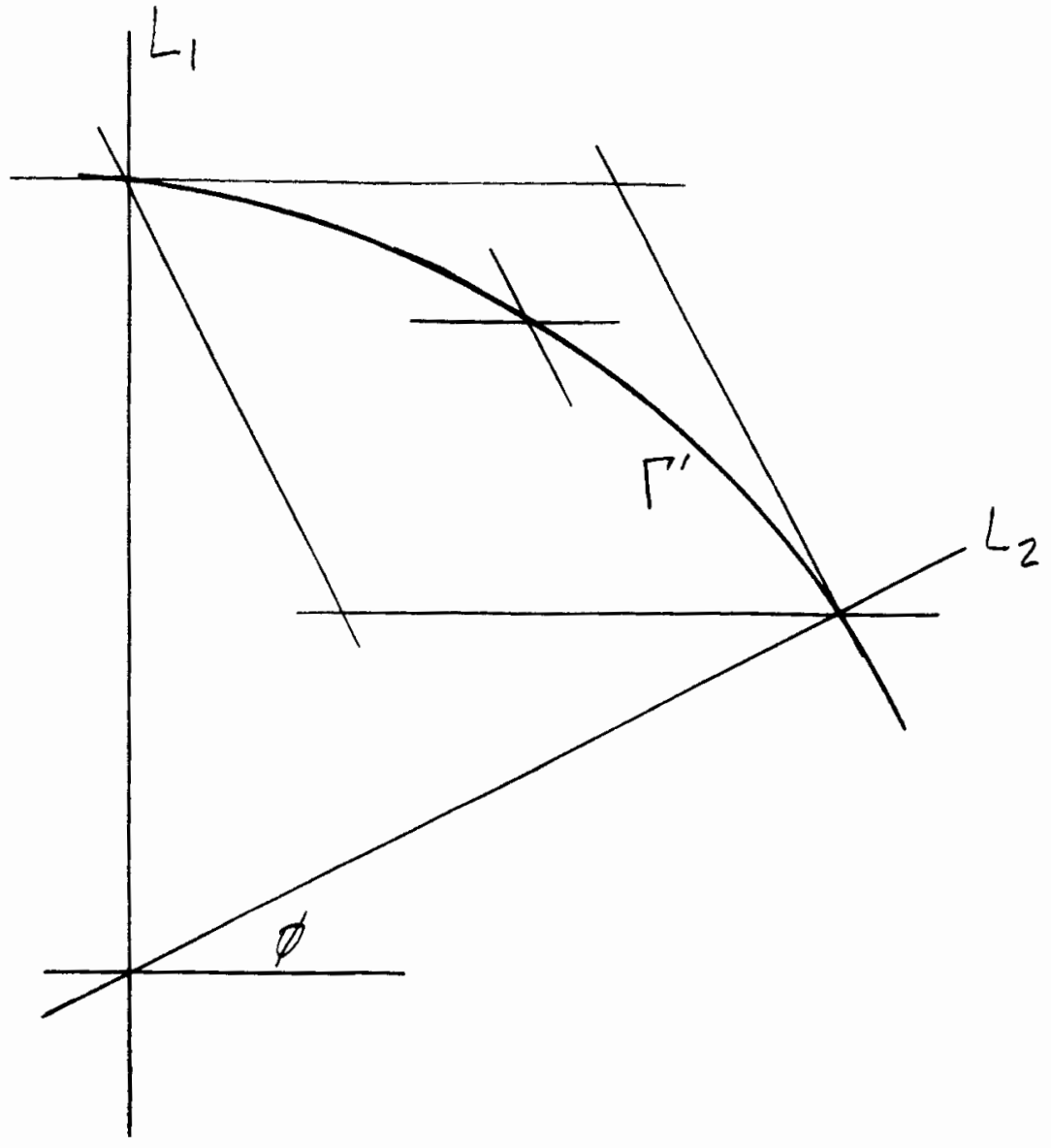

Figure 1. $\Gamma^{\prime}$ is the graph of a decreasing function. 


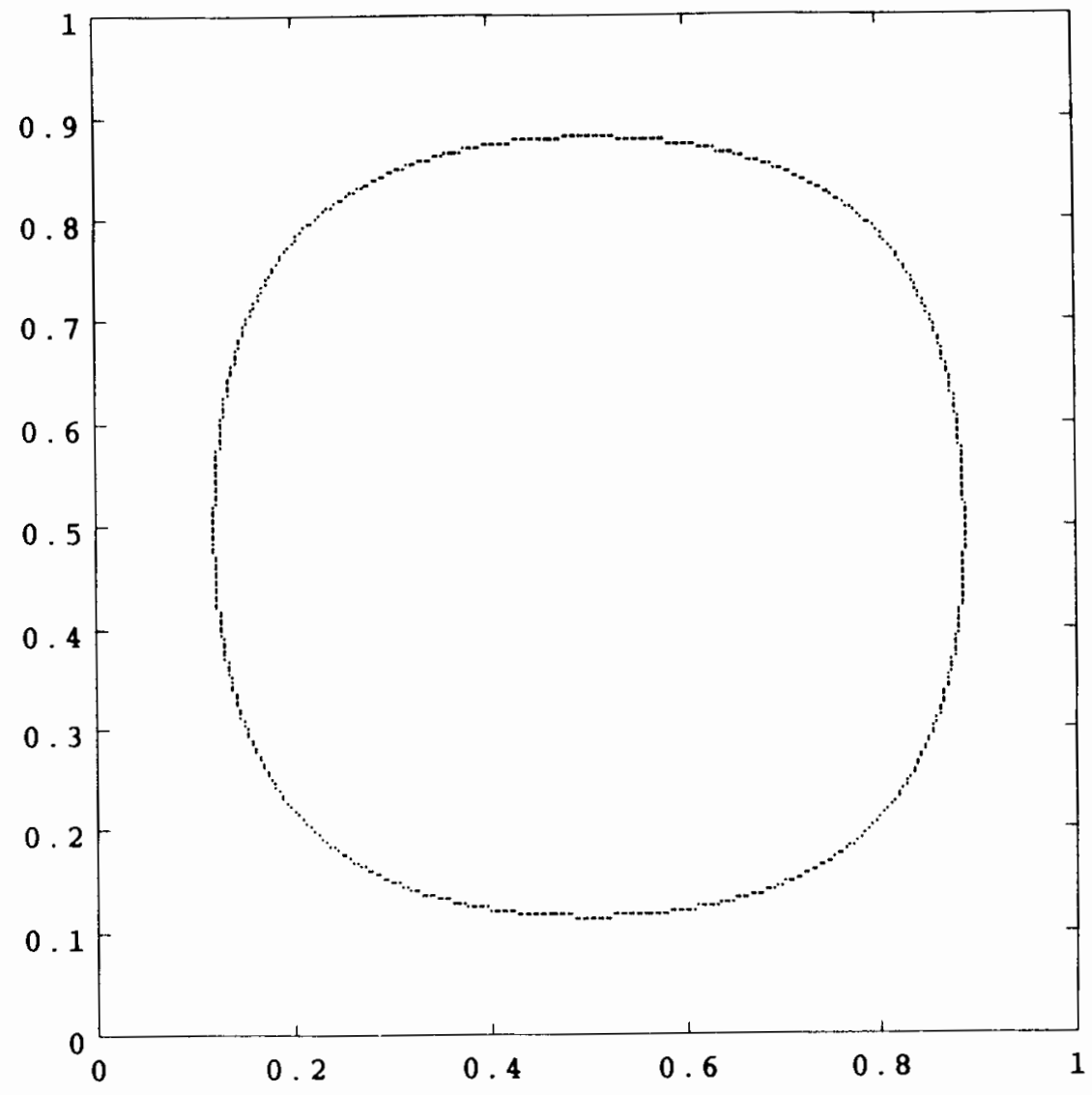

Figure 2. Two phase square drum with deepest bass note. $\alpha=1, \beta=9, \gamma=1 / 2$.

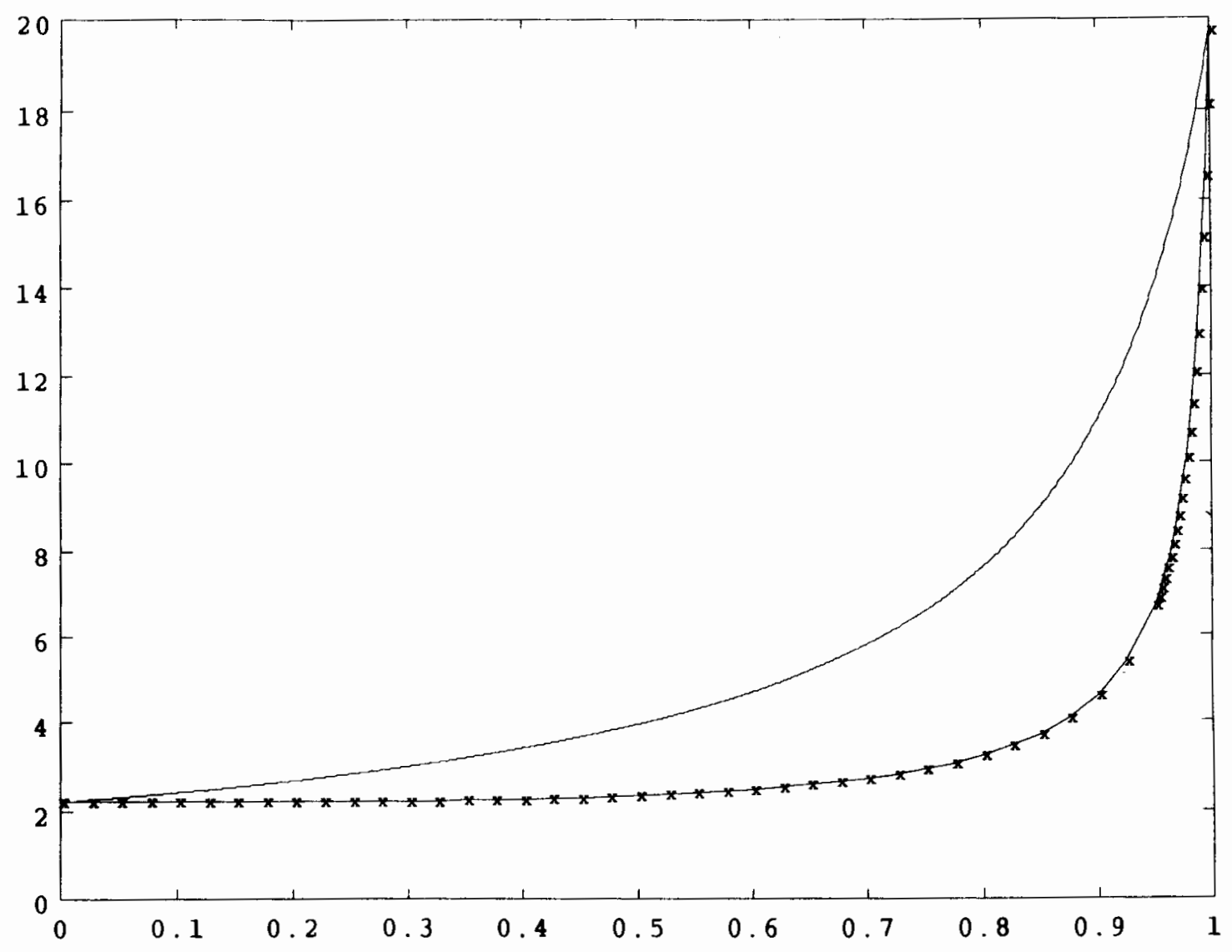

Figure 3. Upper curve: Least eigenvalue of the homogeneous square drum of mass $\gamma+9(1-\gamma)$ vs. $\gamma$. Lower curve: Minimum least eigenvalue of square two phase drum with densities 1 and 9 in area fractions $\gamma$ and $1-\gamma$ vs. $\gamma$. 


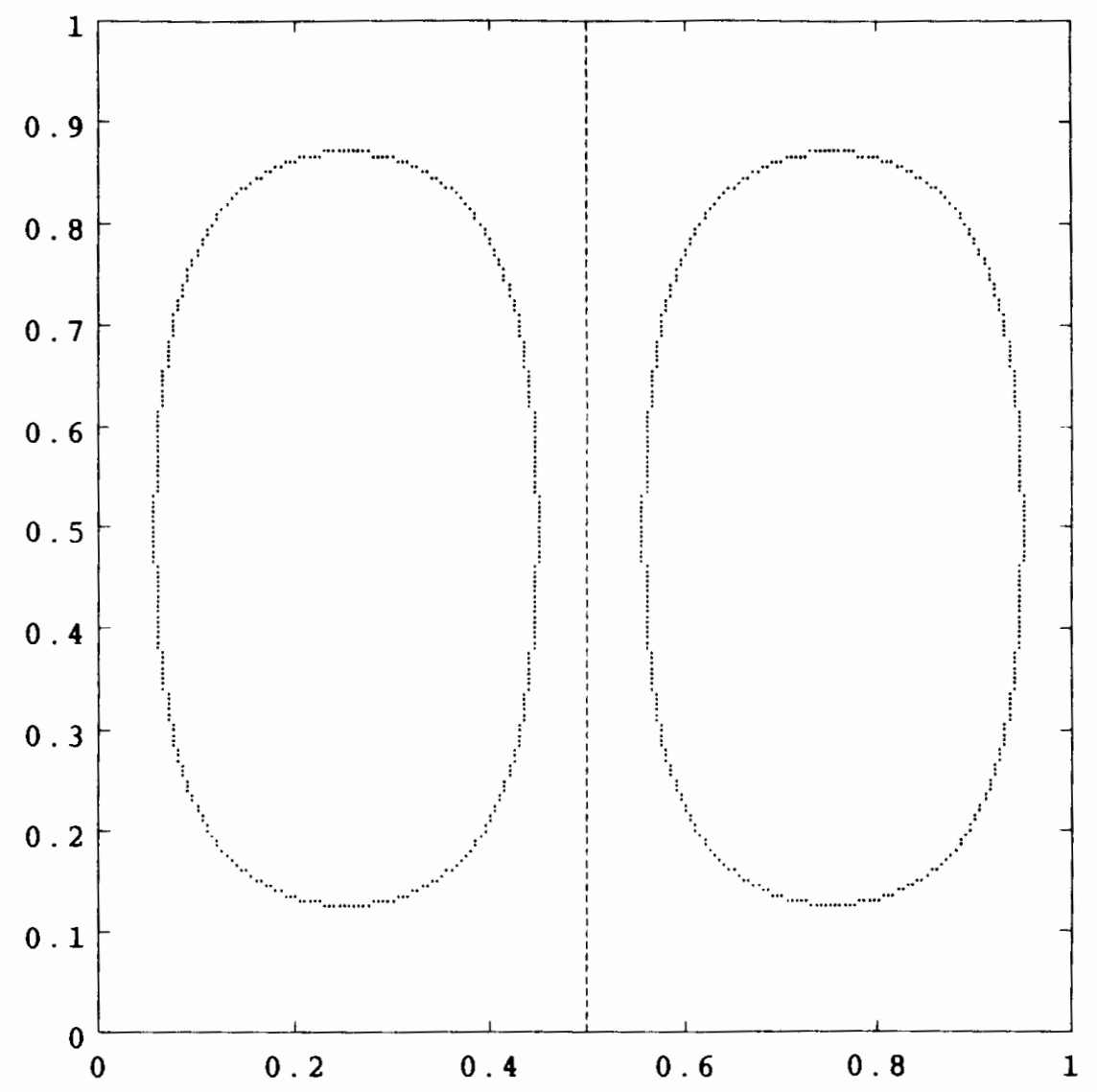

Figure 4. Stationary point of the algorithm applied to $\rho \mapsto \lambda_{2}(\rho)$ on a square drum with $\alpha=1, \beta=9, \gamma=1 / 2$.

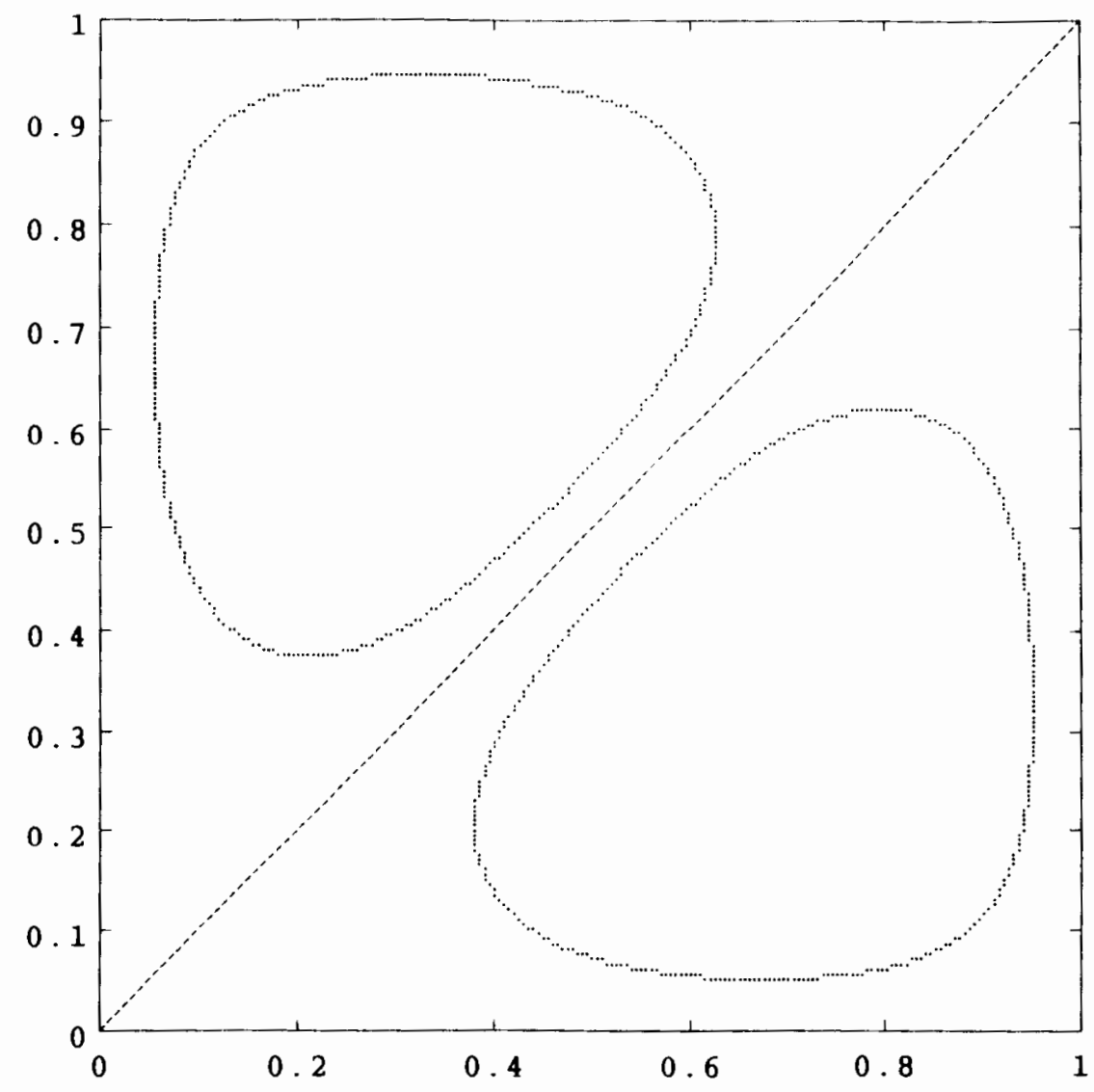

Figure 5. Stationary point of the algorithm applied to $\rho \mapsto \lambda_{2}(\rho)$ on a square drum with $\alpha=1, \beta=9, \gamma=1 / 2$. 